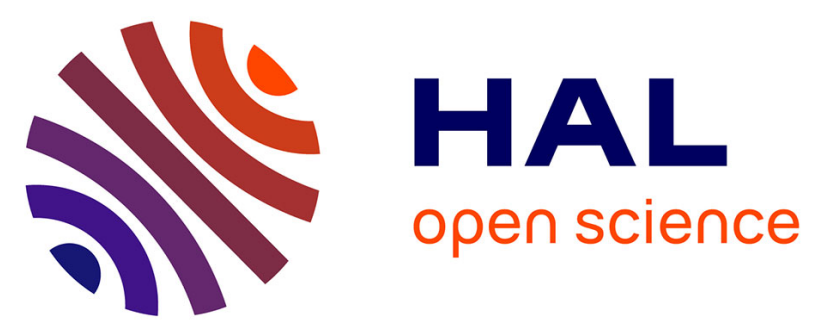

\title{
TiNxOy/TiN dielectric contrasts obtained by ion implantation of ; structural, optical and electrical properties
}

E Punzón Quijorna, V Torres Costa, F Agulló-Rueda, P Herrero Fernández, A Climent, F Rossi, M Manso Silván

\section{To cite this version:}

E Punzón Quijorna, V Torres Costa, F Agulló-Rueda, P Herrero Fernández, A Climent, et al.. TiNxOy/TiN dielectric contrasts obtained by ion implantation of ; structural, optical and electrical properties. Journal of Physics D: Applied Physics, 2011, 44 (23), pp.235501. 10.1088/0022$3727 / 44 / 23 / 235501$. hal-00623796

\section{HAL Id: hal-00623796 https://hal.science/hal-00623796}

Submitted on 15 Sep 2011

HAL is a multi-disciplinary open access archive for the deposit and dissemination of scientific research documents, whether they are published or not. The documents may come from teaching and research institutions in France or abroad, or from public or private research centers.
L'archive ouverte pluridisciplinaire HAL, est destinée au dépôt et à la diffusion de documents scientifiques de niveau recherche, publiés ou non, émanant des établissements d'enseignement et de recherche français ou étrangers, des laboratoires publics ou privés. 
$\mathrm{TiN}_{\mathrm{x}} \mathrm{O}_{\mathrm{y}} / \mathrm{TiN}$ dielectric contrasts obtained by ion implantation of $\mathrm{O}_{2}{ }^{+}$; structural, optical and electrical properties.

E. Punzón Quijorna ${ }^{1,2}$, V. Torres Costa $^{1}$, F. Agulló-Rueda ${ }^{3}$, P. Herrero Fernández ${ }^{3}$, A. Climent $^{1,2}$, F. Rossi ${ }^{4}$, M. Manso Silván ${ }^{1}$.

1. Departamento de Física Aplicada. Universidad Autónoma de Madrid, 28049 Madrid, Spain.

2. Centro de Microanálisis de Materiales, Universidad Autónoma de Madrid, 28049 Madrid, Spain.

3. Instituto de Ciencia de Materiales de Madrid, CSIC, Cantoblanco, 28049 Madrid, Spain.

4. Institute for Health and Consumer Protection, Joint Research Center, 21020 Ispra (Va), Italy.

\section{Abstract:}

The properties of TiN can be gradually transformed by $\mathrm{O}_{2}{ }^{+}$implantations in the 10- 40 $\mathrm{KeV}$ range and fluences in the $5.10^{13}-5.10^{16} \mathrm{~cm}^{-2}$ range. The resulting structure consists of shallow $\mathrm{TiN}_{\mathrm{x}} \mathrm{O}_{\mathrm{y}}(\mathrm{TiNO}) / \mathrm{TiN}$ contrasts with increased resistivity on the top layer. In fact, oxygen actively replaces nitrogen in the implanted TiN region as illustrated by Rutherford Backscattering Spectrometry. N substitutions and vacancies in the lattice induce structural distortions and strain generation as illustrated by X-ray diffraction, high resolution transmission electron microscopy and Raman spectroscopy. The influence of these modifications in the optical and electrical properties was characterized by spectroscopic ellipsometry and four probe resistivity measurements. The proposed process, especially at the lower energy, is liable to help in the creation of electrical/photonic structures based in shallow TiNO/TiN electric/dielectric contrasts.

Keywords: TiN, oxygen implantation, electric/dielectric contrasts, HR-TEM. 


\section{Introduction}

TiN is a reputed material exploited in the fields of electronics [1], optics [2,3] and hard-anticorrosive thin films $[4,5]$. With regard to the former, the good electrical conductivity of $\mathrm{TiN}$ oriented to its application as diffusion barrier for $\mathrm{Cu}$ contacts in very large scale integration for microelectronics [1]. Optical studies on TiN have been mainly an accurate resource to determine the dielectric function of TiN preparations [2] or describing its plasmonic properties [3]. In fact, the fabrication of micro-nano structured TiN materials in the appropriate matrix would allow the exploitation of plasmonic devices [6].

The search for adapted TiN properties for specific applications opened the path for the proposal of derived ternary compounds among which, TiCN [7] and TiBN [8] for tribomechanics, TiAlN for corrosion resistance [9], TiCrN for magnetic properties [10] or TiNO [11,12] for modified optical properties. The exploitation of TiN in plasmonic devices passes through structural requirements such as patterning in dielectric micro and nano environments [6]. In this frame, an accurate localized transformation of TiN into TiNO could be an attractive way to produce functional electric-dielectric electromagnetic bandgap structures [13]. However, processing challenges arise in the preparation of such structures. The controlled growth of TiNO is extremely difficult in view of the drastic integration of oxygen in the structure when mixed plasmas are used [14,15] or unexpected $\mathrm{O}$ sources are present in the chamber [11] (e.g. very low $\mathrm{O}_{2}$ partial pressures lead to $\mathrm{O}$ rich TiNO). Modification approaches have also been proposed; anodic oxidation [16] or O plasma immersion methods [12] were described as potential processes to avoid thermal modification, which produces extended diffusion profiles of oxygen that limit the possibility of creating structured domains [17]. Bearing in mind the stability against ion mixing of TiN films bombarded in the $100 \mathrm{KeV}$ range [18], in this work we evaluate the possibility of producing 
modification of electronic and optical characteristics of TiN by $\mathrm{O}_{2}{ }^{+}$ion implantation to give rise to sharp TiNO/TiN interfaces.

\section{Materials and Methods}

2.1 Materials processing and modification.

TiN films used for ion implantation in this work were obtained by reactive magnetron sputtering in an Ion Tech sputtering system. The deposition pressure was $0.2 \mathrm{~Pa}$ and a power of $300 \mathrm{~W}$ DC was applied to the magnetron, which contained a high purity $\mathrm{Ti}(99.995 \%)$ target within a mixed argon-nitrogen discharge $(\mathrm{Ar} / \mathrm{N}=9 / 1)$. A steady atmosphere was reached by 30 min pre-sputtering with the reactive gas mixture attaining growth rates of $200 \mathrm{~nm} / \mathrm{h}$. Substrates were $\mathrm{Si}(100)$ or thermal $\mathrm{SiO}_{2} / \mathrm{Si}(100)$ depending on the characterization technique envisaged. Final TiN film thicknesses was circa 180 and $90 \mathrm{~nm}$ for $\mathrm{Si}(100)$ and $\mathrm{SiO}_{2}$ substrates, respectively.

The implantation of $\mathrm{O}$ ions into the TiN films was simulated by using the "Stopping and Range of Ions in Matter" (SRIM) software code [19]. Simulation of atomic O implantations in spite of the molecular $\mathrm{O}_{2}{ }^{+}$helps in determining a maximum in the penetration depth. The process does not take into account the broad spectrum of distribution energies for the dissociated $\mathrm{O}$ atoms induced upon interaction of the molecular $\mathrm{O}_{2}{ }^{+}$beam with the TiN sample during the experiment. The results are presented considering a stoichiometric 1:1 TiN. After selection of the energy range (1-40 KeV), simulations were performed using the "Surface Sputtering" calculation for 10000 ions. Simulations were performed to estimate the implantation depth and to illustrate the distribution of atoms at the first stages of implantations. The TiN coatings were implanted with $\mathrm{O}_{2}{ }^{+}$in a DanFysik high current ion implanter. Irradiation at energies of 10,20 and $40 \mathrm{KeV}$ (samples labeled E1, E2 and E3 respectively) and fluences of $5.10^{13}, 10^{15}$ or $5.10^{16} \mathrm{~cm}^{-2}$ (samples labeled F1, F2 and F3, 
respectively) was carried out by cooling at room temperature and a chamber operation pressure of less than $10^{-5} \mathrm{~Pa}$.

\subsection{Materials Characterization.}

Rutherford backscattering spectrometry (RBS) was performed at the Centro de MicroAnálisis de Materiales-Universidad Autónoma de Madrid (Spain) using the $5 \mathrm{MeV}$ tandetron accelerator [20]. Measurements were performed with a $3.035 \mathrm{MeV} \mathrm{He}^{+}$beam to exploit the resonance of the ${ }^{16} \mathrm{O}(\alpha, \sigma){ }^{16} \mathrm{O}$ elastic scattering cross section. The incidence angle with respect to the surface normal was $74^{\circ}$, and the scattered particles where detected at an angle of $170^{\circ}$ by using a silicon surface barrier detector. The experimental spectra have been fitted for quantitative purposes by using the SIMNRA code [21] to determine the film structure.

X-Ray diffraction (XRD) was performed in a Siemens diffractometer using $\theta / 2 \theta$ configuration with $0.02^{\circ}$ scan step and $6 \mathrm{~s}$ integration time. The presence of strain in the modified samples was obtained from the analysis of the diffractograms using the Stokes Wilson formula [22]. Raman spectra were measured with a Renishaw Ramascope 2000 spectrometer and an argon ion laser (operating at a wavelength of $514.5 \mathrm{~nm}$ ). Laser power on the sample was around $3 \mathrm{~mW}$. An optical microscope (100× objective) was used to focus the laser light on the sample and to collect the scattered light in a backscattering geometry. The system was configured in a quasi-confocal mode. No polarization analyzer was used before the spectrometer.

Cross-sectional samples (TiN reference and TiNE1F3 samples) for transmission electron microscopy (TEM) were prepared by dimple-grinding with a Gatan 656 dimpler and ion-milling with a Fischione 1010 as previously described [23]. Transmission electron microscopy studies were done in a Philips Tecnai 20F FEG analytical microscope operating at $200 \mathrm{kV}$ and equipped with a dark field high angle annular detector (HAAD) for Z-contrast 
analysis. Electron diffraction patterns were obtained by the selected area method (SAED). limage processing of the high-resolution images was carried out using Gatan Digital Micrograph and ImageJ software packages.

Four probe measurements were performed in a home made system located at the microelectronics laboratory (UAM) by scanning currents from -100 to $100 \mathrm{~mA}$. In this range all the samples showed an ohmic linear regime. Sheet resistance $\left(\mathrm{R}_{\mathrm{s}}\right)$ values were obtained considering a correction factor of $\mathrm{CF}=4.2357$ [24]. Spectroscopic Ellipsometry (SE) Measurements were performed in a FLS-300 (JA Woollam Co., Inc) using a low power illumination at fixed angle $\left(72,5^{\circ}\right)$ and covering the spectral range of $250-1800 \mathrm{~nm}$ with simultaneous detection by using a CCD.

\section{Results and discussion}

\section{$3.1 \mathrm{O}_{2}^{+}$implantation.}

Implantation conditions for $\mathrm{O}_{2}{ }^{+}$ions were selected on the basis of simulation of $\mathrm{O}$ ion implantations that allow predicting a maximum in the penetration depth of $\mathrm{O}$. It is considered that molecular oxygen will dissociate upon interaction with the sample distributing the energy between the two dissociated atoms (not in identical parts). Distributions are only considered to illustrate the damage induced at the first stages of implantation (i.e. disregarding accumulated damage). The corresponding $\mathrm{O}$ ion and recoil ionization profiles generated by SRIM (mainly $\mathrm{N}$ recoils) (Figure 1.a) illustrate that, at energies between 10 and $40 \mathrm{KeV}$, the incident ions easily generate recoils, which become active species in producing considerable damage by nuclear energy loss. In fact, at the range of depths in this study, the energy released by recoil ionization corresponds to a notable fraction of that released by incident $\mathrm{O}$ ions (circa $2 / 3,1 / 2$ and $1 / 3$ for 10,20 and $40 \mathrm{KeV}$ implantation respectively). Note also that at $20 \mathrm{KeV}$ a maximum is reached in the energy released per $\mathrm{O}$ ion, which justifies the selection of the 
energy ranges in this study. Furthermore, these ionization events can be induced up to depths of $100 \mathrm{~nm}, 60 \mathrm{~nm}$ or $30 \mathrm{~nm}$ for implantation energies of 40, 20 or $10 \mathrm{KeV}$, respectively. By looking at the final ion and recoil in depth distribution (figure 1.b), one observes that a very intense relocation of recoiled atoms is induced (see two order of magnitude scale correction between ion and recoil axis) with a maximum and attenuation of distribution at depths related directly to those mentioned previously for ionization energy. On the other hand, $\mathrm{O}$ ions accumulate considerably deeper than recoils with concentration maxima at depths much deeper than those where ionization damage occurs (i.e. for $40 \mathrm{KeV}$, incident $\mathrm{O}$ ionization damage maximum takes place at circa $10 \mathrm{~nm}$ while final $\mathrm{O}$ distribution maximum appears at circa $50 \mathrm{~nm}$ ). It can be also observed that the higher the implantation energy, the broader the final $\mathrm{O}$ ion distribution. These considerations are important in order to compare with experimental data obtained by the subsequent multi-technique analysis but should be improved by alternative simulations to describe with more precision the dynamic changes in the composition due to accumulated damage [25].

\subsection{Oxygen integration.}

The in-depth composition and degree of $\mathrm{O}$ incorporation in the TiN/Si(100) films was probed by non Rutherford RBS (O resonance with $3.035 \mathrm{KeV} \mathrm{He}^{+}$beam) to enhance $\mathrm{O}$ sensitivity. Data were obtained for the reference TiN sample and the TiNO samples produced at different energies for a fixed intermediate fluence (Figure 2.a) and different fluencies for the lower energy (Figure 2.b). The fitting of the spectra corresponding to the control sample indicates that the original TiN films contained already $\mathrm{O}$ (up to 3 at. \%). However, previous studies suggest that $\mathrm{O}$ adsorbs along TiN crystal boundaries upon atmospheric exposure [26] leading to a base $\mathrm{O}$ concentration of circa $3 \%$ as established by RBS [27]. Spectra corresponding to modified TiNO films were simulated according to a simplified three layer 
model (see continuous line spectra in figures 2.a and 2.b). Total thickness after implantation was conserved so that sputtering effects could be considered as negligible at the energy and fluence ranges considered in this work. This is in agreement with what is observed by RBS characterization of anodically oxidized TiN [16] and results in a clear advantage with respect to the oxidation induced by plasma immersion under bias potential [12], which induces considerable layer sputtering.

From the analysis of data corresponding to the sweep in $\mathrm{O}_{2}{ }^{+}$implantation energy, it can be observed that different energies gave rise to oxygen containing layers with thicknesses of circa 35, 50 and $75 \mathrm{~nm}$ for E1, E2 and E3, respectively. For a given fluence (figure 2.a), results comply with the rule foreseen by simulation results: the lower the implantation energy, the lower the implantation depth but the higher the $\mathrm{O}$ concentration (note inset with magnified $\mathrm{O}$ peak intensity). An increase of fluence produces the expected increase in the $\mathrm{O}$ concentration. However, it should be mentioned that the distribution of $\mathrm{O}$ does not exactly reproduce the simulated distributions (not even in the low implantation regime). This is related to the fact that simulations are performed with single atom ions, while experimental implantations are performed with $\mathrm{O}_{2}^{+}$, which drastically enlarges the distribution energy of $\mathrm{O}$ when reaching the surface giving rise to considerable $\mathrm{O}$ concentration on the surface.

With regard to the aims of this work, increased surface contrasting properties are obtained for samples with lower energy implantation and higher fluence. It is important to remark that the surface composition of this particular sample presents the stoichiometry of a material closer to $\mathrm{TiO}_{2}$ than to $\mathrm{TiN}\left(\mathrm{TiN}_{0.51} \mathrm{O}_{1.26}\right)$. This stoichiometry indicates the presence of extra $\mathrm{N}$ and $\mathrm{O}$ with respect to what would be expected for derived Ti compounds.

\subsection{Structural Modification.}


The XRD diagrams corresponding to $\mathrm{TiN} / \mathrm{Si}(100)$ and $\mathrm{O}_{2}{ }^{+}$implanted $\mathrm{TiN} / \mathrm{Si}(100)$ are presented in Figures 3.a and 3.b for implantations at increasing energy and increasing fluence, respectively. All the films present the characteristic peaks of TiN (JCPDS 38-1420) with a diffraction peak intensity distribution that was slightly modified as a function of the $\mathrm{O}_{2}^{+}$ implantation treatment. In the reference TiN sample, the peaks corresponding to (111) planes presented a remarkably higher intensity/width ratio than those corresponding to (200) planes (diffraction maximum reference). This indicates a texturization previously observed in TiN films grown by magnetron sputtering [28], which can be optimized in controlled conditions [29]. The diffraction diagrams for implanted samples presented no new peaks but notable distortions of peak intensity/width ratios and peak position shifts with respect to the TiN reference. In fact, damage induced by implantation is known to induce such shifts related to a slight reduction of lattice parameter. From Figure 3.a it can be observed that the higher the implantation energy, the closer the diffraction pattern to the one of reference TiN. This feature is a clear indication that the damage is produced by accumulation of oxygen rather than by direct impact damage during irradiation. This is in agreement with the fact that simulated ionization energy is similar for the three energies studied. Clearly, for a given implantation energy (see i.e. for $\mathrm{E}=10 \mathrm{KeV}$ in figure 3.b) the higher the dose, the higher the deviation of the diffraction pattern from that of the reference. These distortions can be interpreted in terms of damage accumulation in the form of stress in the films.

In order to estimate such modifications, the Stokes Wilson formula has been used to determine the strain for the most relevant modification at E1 (figure 4). It can be derived from these data that, especially at the 111 direction, that the higher the implantation fluence, the higher the strain introduced in the modified layer. In fact, the widening of diffraction peaks can be attributed to the modification of lattice parameters with respect to the original cell. It is worth noting that, though the modified layer reaches a composition of $\mathrm{TiN}_{0.51} \mathrm{O}_{1.26}$, the 
structure remains face centered cubic with a lattice parameter close to that of TiN. This reveals the relevant tightening effect of the unmodified TiN film below since relevant structural changes have been described for $\mathrm{TiN}_{0.74} \mathrm{O}_{0.36}$ and $\mathrm{TiN}_{0.6} \mathrm{O}_{0.4}$ (JCPDS files 00-049-1325 and 00044-0951, respectively), which contain a considerably lower structural O. These data point out to the presence of considerable stress fields in the implanted regions. Further insight into this phenomenon can be extracted from the estimations of lattice parameters. The results indicate that the cell tends to stretch, which is compatible with a reduction of $\mathrm{N}$ in the crystals [30]. These new data reveal that 111 planes are preferentially affected and become closer after modification. This lattice distortion in the modified layer correlates with the creation of a strain field at the interface with the native TiN.

In order to get an insight into the local structure of the irradiated areas a TEM study was performed on samples treated at the lower energy and higher dose (E1F3) to compare with $\mathrm{TiN} / \mathrm{SiO}_{2} / \mathrm{Si}(100)$. The general view of the reference and irradiated structures can be seen in figure 5.a and 5.b, respectively. The TiN films of $90 \mathrm{~nm}$ thickness present the typical columnar growth on the $\mathrm{SiO}_{2}$ substrate, as previously observed for $\mathrm{Si}$ substrates [28]. The columnar finishing gives rise to an irregular surface with an estimated roughness of circa 5 nm. The implanted TiN sample presents a layered structure consisting of an amorphised top film (of approximately $20 \mathrm{~nm}$ ) and a relatively unaltered TiN transition layer. The external surface truncates thus the columnar structure of the underlying TiN and allows the formation of a much more homogeneous surface. The total thickness of the structure remains constant so that sputtering processes during implantation can be considered as negligible.

HR TEM images were obtained from the $\mathrm{SiO}_{2} / \mathrm{TiN}$ interfaces and from the outer TiNO irradiated areas. Figure 5.c shows the interface between the underlying $\mathrm{SiO}_{2}$ and the non irradiated bottom part of the TiN coating from the square inset in figure 5.b. Several crystalline structures with different orientations (resolved in 2D or 1D) corresponding to the 
TiN columnar growth can be clearly identified. The polycrystalline structure is confirmed by the Fourier transformed of the area corresponding to TiN (see inset pattern in image 5.c), which shows a multi-domain diffraction pattern of relatively intense dots. Figure 5.d presents the HR TEM image of the outer TiNO resulting from irradiation, as extracted from the inset in figure 5.b. A transition line to the gluing material of the preparation has been drawn to guide the eye. The image confirms the slight roughness reduction of the irradiated surface with respect to the original columnar structures (mean roughness of circa $2.5 \mathrm{~nm}$ ). The remaining of partially crystalline structure (i.e. only partial amorphization takes place during irradiation) can be confirmed both by the presence of resolved planar distances and the dot patterns obtained by the Fourier analysis of the TiON areas in the image (inset to figure 5.d). However, dot radial analysis does not confirm such structures correspond to the same TiN dots observed in the Fourier transformed image of TiN in figure 5.c.

To deepen our understanding of the microstructure of the irradiated films we have also measured the Raman spectra for various irradiation energies and fluencies on $\mathrm{TiN} / \mathrm{Si}(100)$ samples. The largest variation is found for the lowest energy E1 because the Raman spectrum is more sensitive to the upper layers due to the light absorption in the material (for higher energies the material is modified at deeper layers). Therefore only the spectra for E1 are shown in Figure 6. The crystal structure of TiN is cubic, with all the atoms at a center of inversion symmetry. Therefore the first order Raman spectrum is forbidden for a perfect crystal. However, the usual presence of nitrogen vacancies and other defects induces some broad band first order Raman spectrum [31]. The non-irradiated TiN film shows main bands at 198 (transverse acoustic phonon, TA), 289 (longitudinal acoustic, LA), 456 (second order, 2A), and $548 \mathrm{~cm}^{-1}$ (transverse optical, TO) [32]. The sharp peak at $520 \mathrm{~cm}^{-1}$ arises from the silicon substrate. 
On irradiation with oxygen ions the TiN Raman bands become more intense with fluence (Figure 6) because the number of defects increases and so does the intensity of the defect-induced first-order Raman spectrum. In particular the acoustic phonon bands become stronger relative to the optical phonon band. Since the latter is at a higher frequency it involves mostly the vibration of the lighter nitrogen atoms, whereas acoustic modes involve mostly vibrations of the heavier $\mathrm{Ti}$ atoms. Therefore the decrease of the TO intensity indicates that the nitrogen content decreases with fluence. The frequencies of the TiN bands increase significantly with fluence (up to $100 \mathrm{~cm}^{-1}$ for the TO phonon), pointing to a shortening of the Ti-N bond distance with irradiation, that correlates to the decrease in the lattice parameter observed by x-ray diffraction. No evidence of new Raman peaks due to transformed phases like $\mathrm{TiO}_{2}$ anatase or rutile has been found. It is interesting to note that the silicon peak intensity increases strongly with irradiation. Since the substrate is buried under the TiNO film, whose thickness does not change with irradiation, we attribute the intensity enhancement to a decrease of the absorption coefficient for the irradiated film, which allows more laser light to reach the substrate and also more scattered light from the substrate to reach the detector. The Raman intensity from the substrate buried under a TiNO film of thickness $d$ is $I=I_{0} \exp (-2 \alpha$ $d$ ), where $I_{0}$ is the laser intensity with no film and $\alpha$ the linear absorption coefficient of the film. From the increase of the silicon Raman intensity and taking $d=180 \mathrm{~nm}$, we estimate a decrease on $\alpha=43000 \mathrm{~cm}^{-1}$ at the laser wavelength $(514.5 \mathrm{~nm})$ for the E1F3 film with respect to the TiN film.

\subsection{Electrical and optical properties}

The modification of the film properties was initially probed by sheet resistance measurements on films deposited on high dielectric constant substrates (TiN/SiO2/Si(100)). The results of these measurements are presented in figure 7 . These resistivity measurements 
indicate that the process of $\mathrm{O}_{2}{ }^{+}$implantation is especially adapted for gradual modifications. Both, variation in energy and fluence produce a scaled increase in the sheet resistance. The results further indicate that the lower energy is better adapted towards production of a deeper electrical modification. For this energy, the highest fluence induces in fact a resistivity value four times higher than the corresponding to the reference $\operatorname{TiN}\left(\mathrm{R}_{\mathrm{s}}=7.7 \Omega /\right.$, in agreement with the sheet resistance measured for TiN films of thicknesses higher than $50 \mathrm{~nm}$ ) [33].

Films with increased contrasting properties (E1 energy implantation) were further characterized by spectroscopic ellipsometry (figure 8). The Psi and Delta curves show that a controlled and scalable modification is indeed obtained by the implantation process. The evolution of the Psi and Delta plots reveal an increasing depolarization with implantation fluence. This depolarizing effect is usually attributed either to an increasing surface roughness or a stronger substrate influence. Since no significant roughness change has been observed on implanted samples, it may be concluded that the increasing depolarization is due to a stronger substrate effect. This result further supports the idea of a decrease in light absorption (i.e. an increase in the transparency of the implanted film) as suggested by the Raman measurements. In an analogue but reversed process, such light absorption decrease is in agreement with the increased absorption in the visible range observed in highly $\mathrm{N}$ doped $\mathrm{TiN}_{2}$ films [34]. In fact, a clear interference effect can be detected in the low wavelength range for the films implanted at the higher fluence (see especially in this case the Delta curve).

\section{Conclusions}

We have described an oxygen implantation process that allows the shallow modification of TiN into $\operatorname{TiN}_{\mathrm{x}} \mathrm{O}_{\mathrm{y}}$. The depth and degree of modification can be tailored by accurately selecting the implantation energy and fluence of implantation. For a determined fluence, implanted oxygen is restricted to a narrower region in depth, giving rise to higher oxygen concentrations 
as demonstrated by RBS. However, the modification is not only an issue of formation of a ternary compound but also the product of intense structural damage in the implanted TiN. Such modification induced partial amorphization of an outer layer and transferred strain to the interface with the underlying TiN. In particular, high resistivity and optical contrasts have been determined by implantations at $10 \mathrm{keV}$ and fluence of $5.10^{16} \mathrm{~cm}^{-2}$, which gave rise to $\mathrm{TiN}_{0.51} \mathrm{O}_{1.26}$ compositions. The outer layer shows increased transparency with respect to the original TiN as illustrated by Raman spectroscopy and ellipsometric measurements. This suggests a neat increase of the dielectric function of the film, which is in agreement with the increase in sheet resistance up to 4 times the value of reference TiN. These results point out to the possibility of growing surface TiNO structures in TiN with potential optical and biomedical applications.

\section{Acknowledgements.}

We acknowledge funding provided by grants MAT2008-06858-C02-01, MAT2008-06858C02-01 and CONSOLIDER-Ingenio FUNCOAT CSD2008-00023 from Ministerio de Ciencia e Innovación (Spain), Madrid Regional Grant "Microseres" and Fundación Domingo Martínez. The technical support from L. García Pelayo and the electrical measurement facilities from the Microelectronics Laboratory (UAM) are greatly appreciated. 
References:

[1] Kohlhase A, Mandl M and Pamler W 1989 J. Appl. Phys. 65 2464-2469.

[2] Fuentes GG, Elizalde E and Sanz JM 2001 J. Appl. Phys. 90 2737-2743.

[3] Hibbins AP, Sambles JR and Lawrence CR 1998 J. Mod. Opt. 45 2051-2062.

[4] Jindal PC, Santhanam AT, Schleinkofer U and Shuster AF 1999 Int. J. Refract. Met. Hard Mater. 17 163-170.

[5] Martin-Palma RJ, Manso M, Martinez-Duart JM, Conde A and Damborenea JJ 2003 J. Vacuum Sci. Technol. A 21 1635-1638.

[6] Cortie MB, Giddings J and Dowd A 2010 Nanotechnology 2111.

[7] Hirsch T and Mayr P 1988 Surf. Coat. Technol. 36 729-741.

[8] Holzschuh H 2004 Thin Solid Films 469 92-98.

[9] Jimenez C, Sanchez-Fernandez C, Morant C, Martinez-Duart JM, Fernandez M and Sanchez-Olias J. 1999 J. Mater. Res. 14 2830-2837.

[10] Mi WB, Ye TY, Wang XC, Tan H and Bai HL 2010 J. Phys. D 4341.

[11] Yokota K, Nakamura K, Kasuya T, Tamura S, Sugimoto T, Akamatsu K, Nakao K and Miyashita F 2002 Surface \& Coatings Technology 158 568-572.

[12] Silvan MM, Rodriguez-Navas C, Gago R, Ruiz JPG and Duart JMM 2008 Surf. Coat. Technol. 203 905-908.

[13] Weily AR, Horvath L, Esselle KP, Sanders BC and Bird TS 2005 Ieee Transact. Anten. Propag. 53 216-223.

[14] Barhai PK, Kumari N, Banerjee I, Pabi SK and Mahapatra SK 2010Vacuum 84 896901.

[15] Braic M, Balaceanu M, Vladescu A, Kiss A, Braic V, Epurescu G, Dinescu G, Moldovan A, Birjega R and Dinescu M 2007 Appl. Surf. Sci. 253 8210-8214.

[16] Montero I, Jimenez C, Albella JM and Perriere J 1993 Surf. Interf. Anal. 20 503-507.

[17] Soriano L, Abbate M, Fuggle JC, Prieto P, Jimenez C, Sanz JM, Galan L and Hofmann S 1993 J. Vac. Sci. Technol. A 11 47-51.

[18] Milosavljevic M, Perusko D, Milinovic V, Stojanovic Z, Zalar A, Kovac J and Jeynes C 2010 J Phys D 436.

[19] Ziegler JF, Ziegler MD and Biersack JP 2010 Nucl. Instrum. Meth. Phys. Res. B 268 1818-1823.

[20] Enguita O, Fernandez-Jimenez MT, Garcia G, Climent-Font A, Calderon T and Grime GW 2004 Nucl. Instrum. Meth. Phys. Res. B 219 384-388.

[21] Mayer M. Application of Accelerators in Research and Industry, Pts 1 and 2 1999: 475: 541-544.

[22] Zhu YH and Li CZ 1997 Mater. Chem. Phy. 51 169-173.

[23] Silvan MM, Langlet M, Duart JMM and Herrero P 2007 Nucl. Instrum. Meth. Phys.

Res. B 257 623-626.

[24] Sze AM. VLSI technology. 2nd edition ed. Mc. Graw Hill, New York, 1988.

[25] Moller W, Eckstein W and Biersack JP 1988 Comp. Phys. Comm. 51 355-368.

[26] Sinke W, Frijilink GPA and Saris FW 1985 Appl. Phys. Lett. 47 471-473.

[27] Bendavid A, Martin PJ, Netterfield RP and Kinder TJ 1996 Surf. Interf. Anal. 24 627-

633.

[28] Pecz B, Frangis N, Logothetidis S, Alexandrou I, Barna PB and Stoemenos J 1995 Thin Solid Films 268 57-63.

[29] Chun JS, Petrov I and Greene JE 1999 J. Appl. Phys. 86 3633-3641.

[30] Kang JH and Kim KJ 1999 J. Appl. Phys. 86 346-350.

[31] Spengler W, Kaiser R and Bilz H 1975 Solid State Comm. 17 19-22. 
[32] Cheng YH, Tay BK, Lau SP, Kupfer H, Richter F 2002 J. Appl. Phys. 92 1845-1849.

[33] Kawamura M, Abe Y and Sasaki K. 1998 J. Vac. Sci. Technol. A 16 200-202.

[34] Chen TL, Hirose Y, Hitosugi T and Hasegawa T 2008 J Phys D 414. 


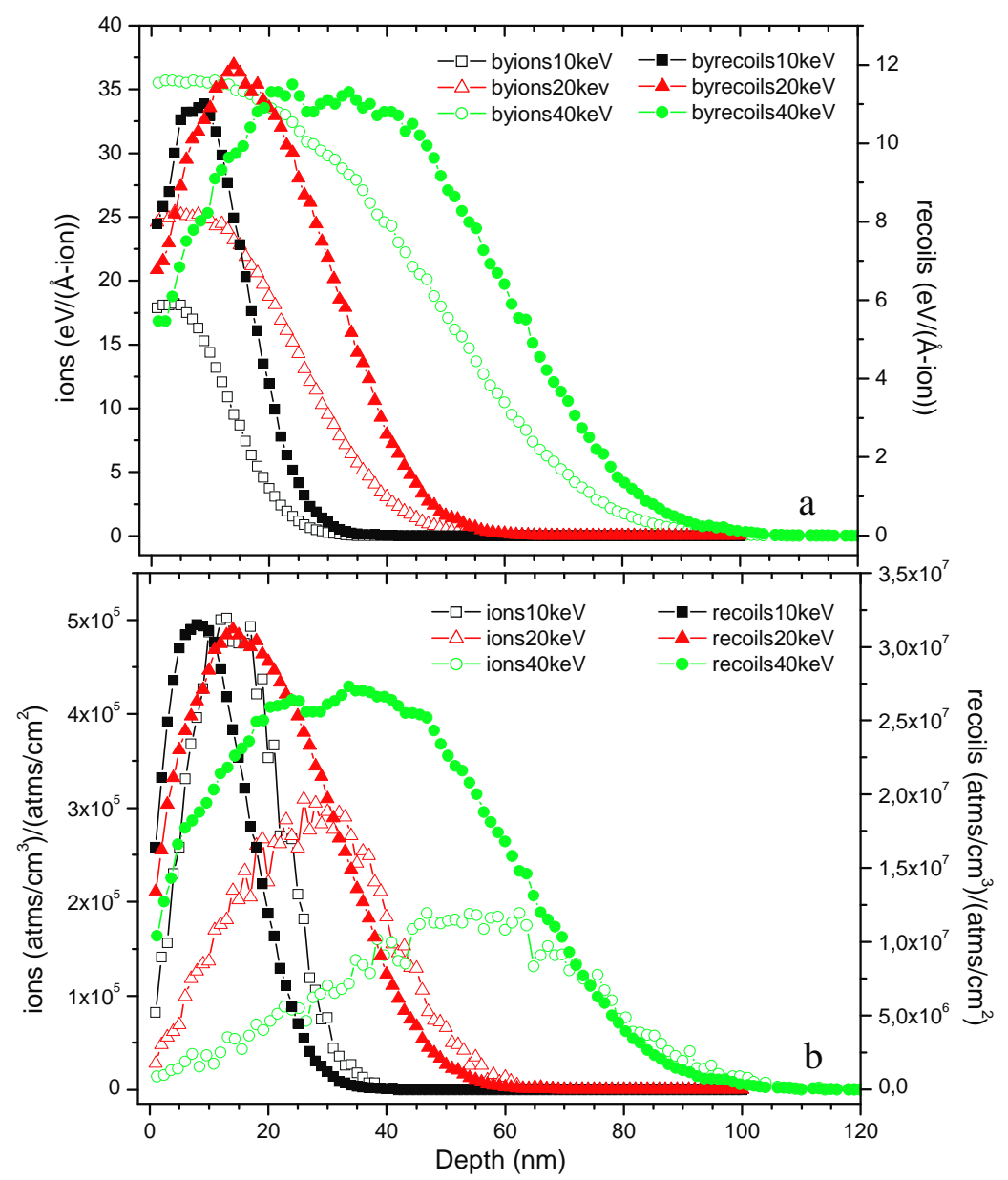

Figure 1: Simulations of oxygen ion and recoil ionization profiles (a) and, oxygen ion and recoil in-depth distribution (b) at energies of 10, 20 and $40 \mathrm{keV}$ (E1, E2 and E3, respectively). 

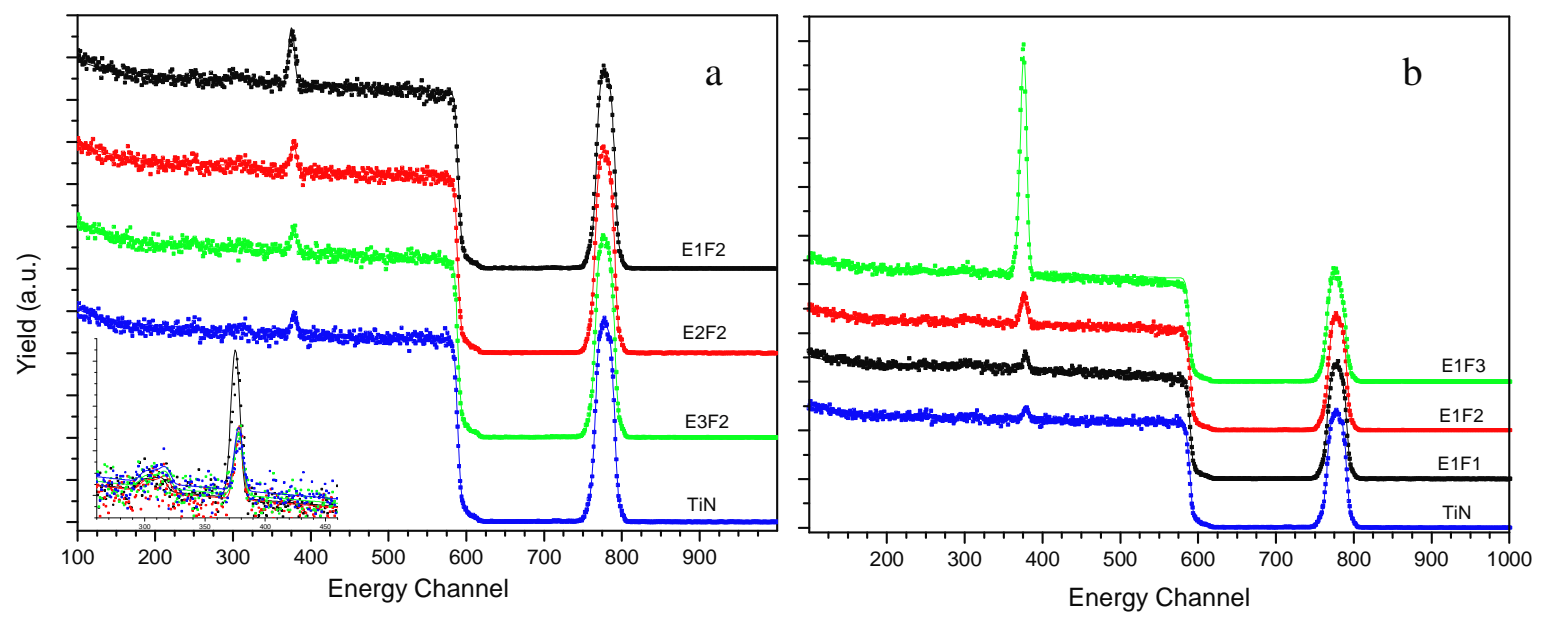

Figure 2: RBS at oxygen resonance $\left(3.035 \mathrm{MeV} \mathrm{He}{ }^{+}\right.$beam) of unmodified sample (TiN/Si), TiNO/Si samples obtained varying energy for the intermediate F2 fluence (a), and varying fluence for the lower E1 energy (b). (Measurement- dot lines, SIMNRA fitting- solid lines). 

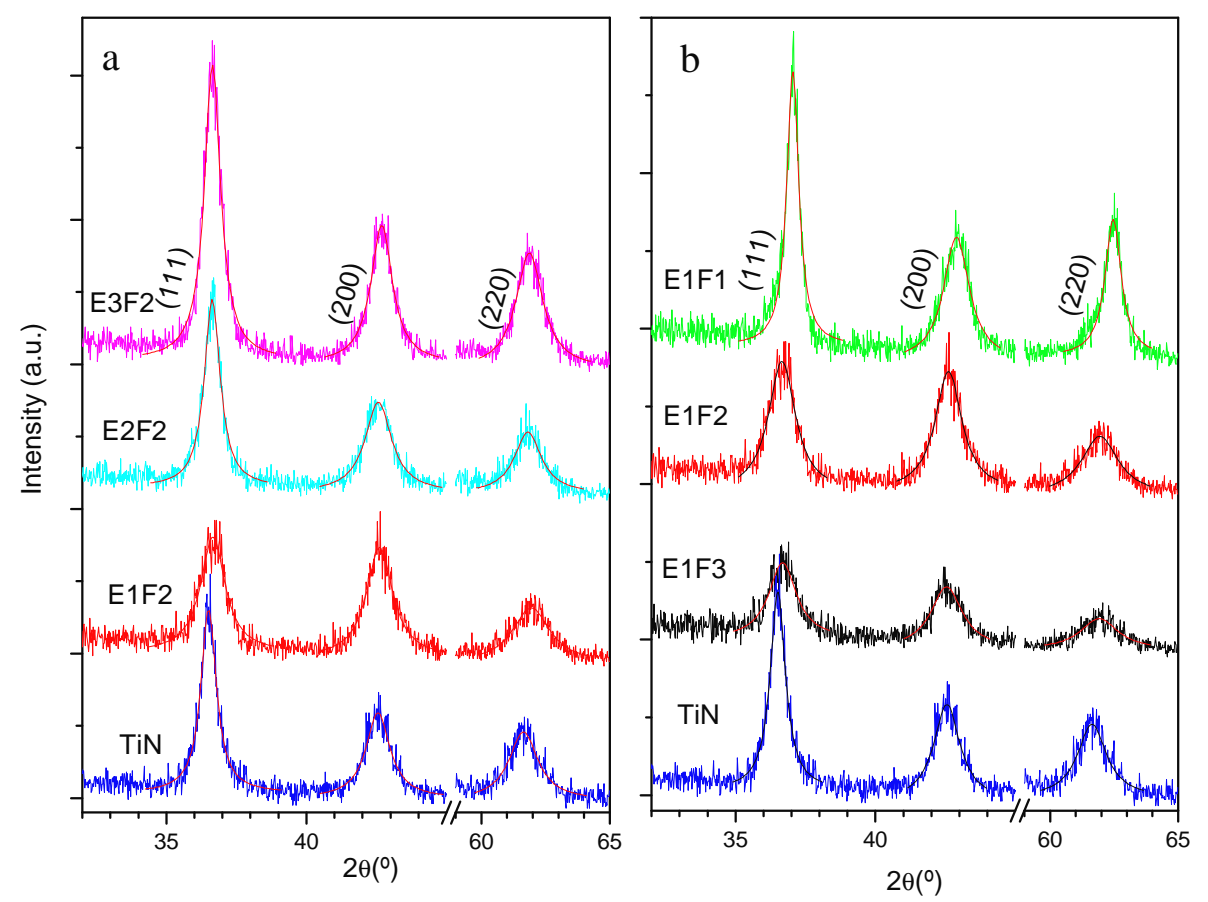

Figure 3: XRD diagrams corresponding to TiN/Si reference and oxygen implanted TiN/Si (100) varying energy for the fixed F2 fluence (a) and varying fluence for the lower E1 energy (b). 


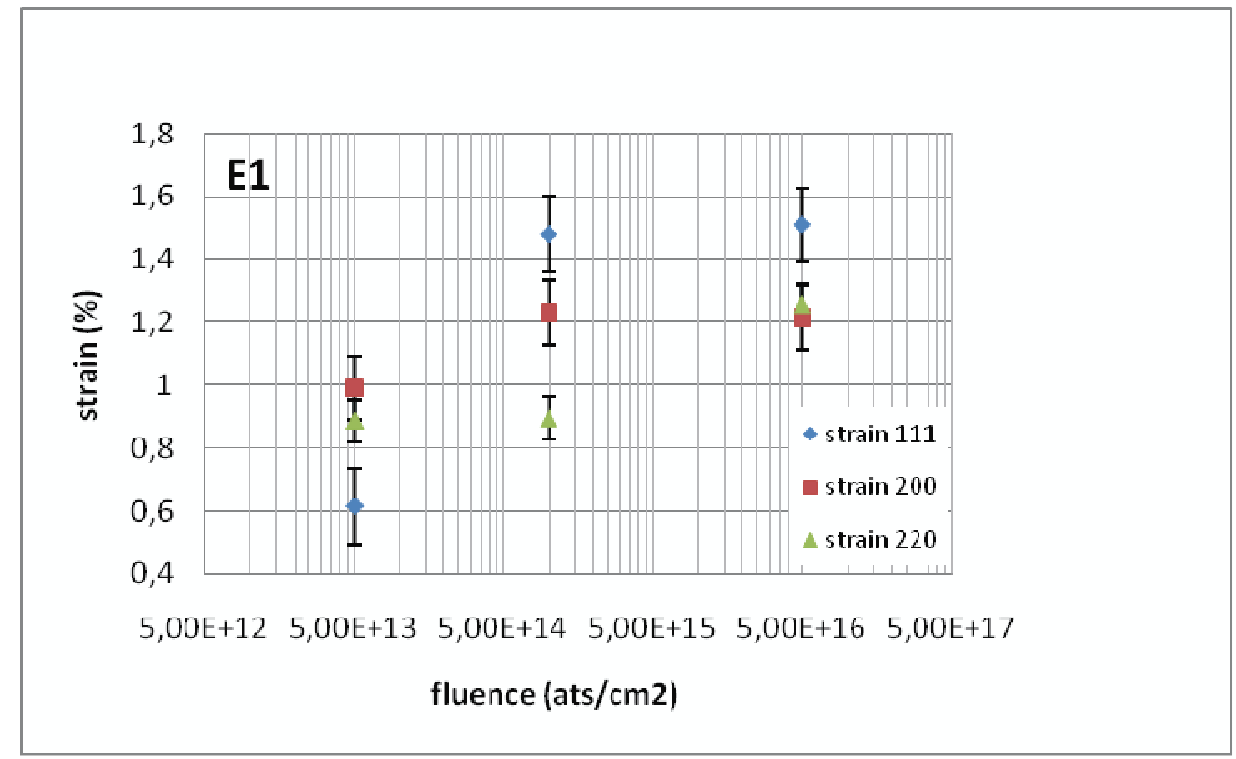

Figure 4: Effect of varying fluence (for the lower E1 energy) in TiN/Si lattice strain calculated from Stokes Willson formula for the different crystal orientations: (111), (200) and (220). 

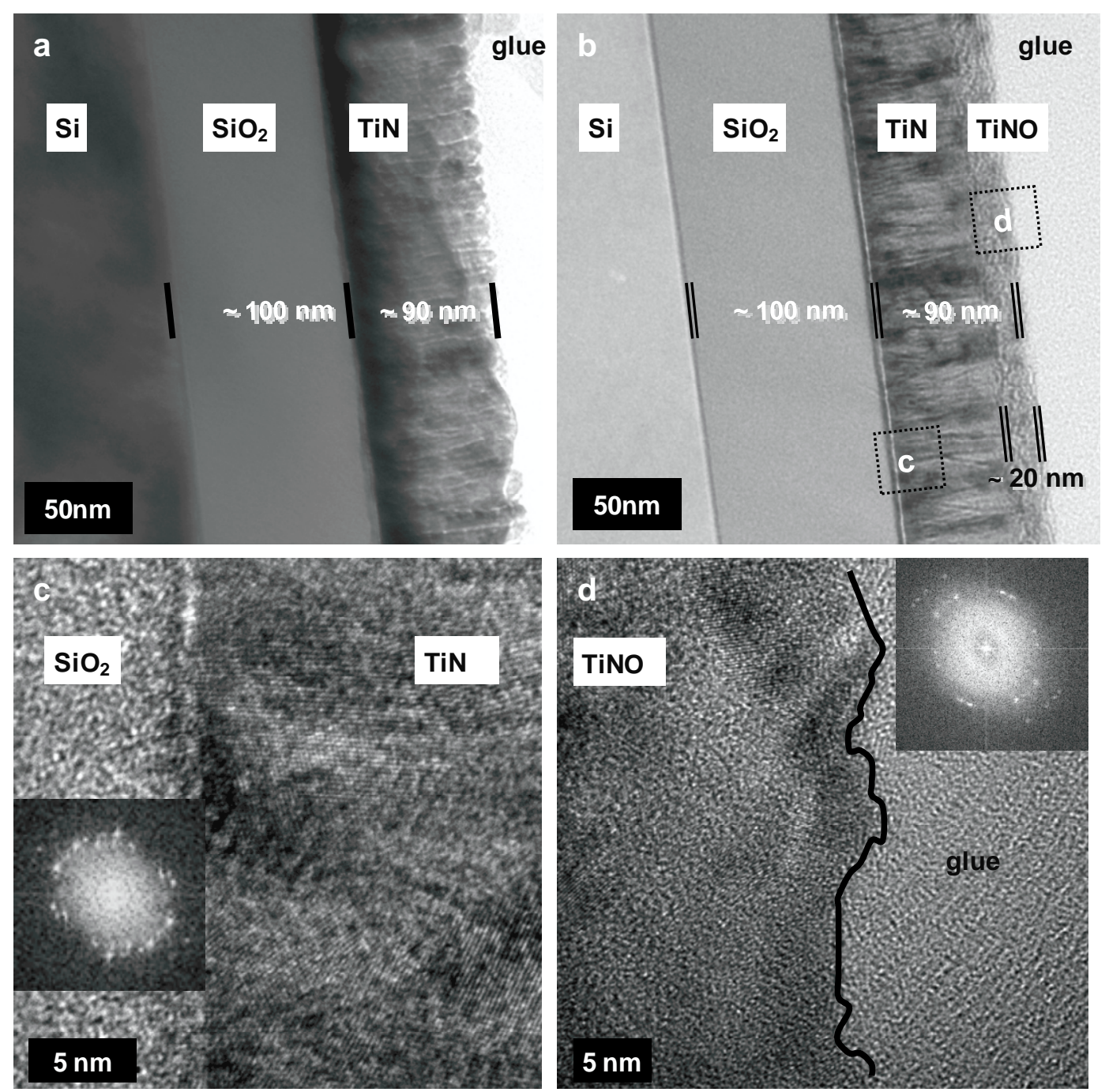

Figure 5. Cross Sectional bright field images of the $\mathrm{TiN} / \mathrm{SiO}_{2} / \mathrm{Si}(100)$ before (a) and after implantation with oxygen at the lower energy (E1) and the highest fluence (F3) (b). HR TEM image from the interface between the $\mathrm{SiO}_{2}$ and $\mathrm{TiN}$ (not in scale) with inset corresponding to the Fourier transformed from the TiN area (c). HR TEM image from the outer irradiated TiNO with inset corresponding to the Fourier transformed from the TiNO area (not in scale) (d). 


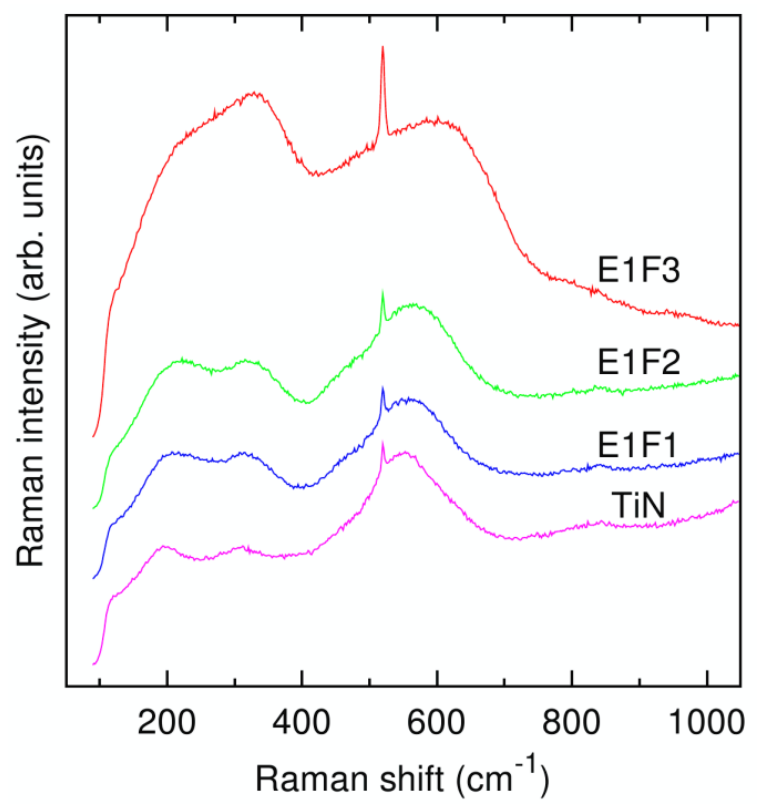

Figure 6: Room temperature Raman spectra of the TiN/Si films before and after irradiation with $10 \mathrm{keV}$ oxygen ions and different fluences (F1, F2, F3). Raman shift relative to the laser excitation wavelength $(514.5 \mathrm{~nm})$. 


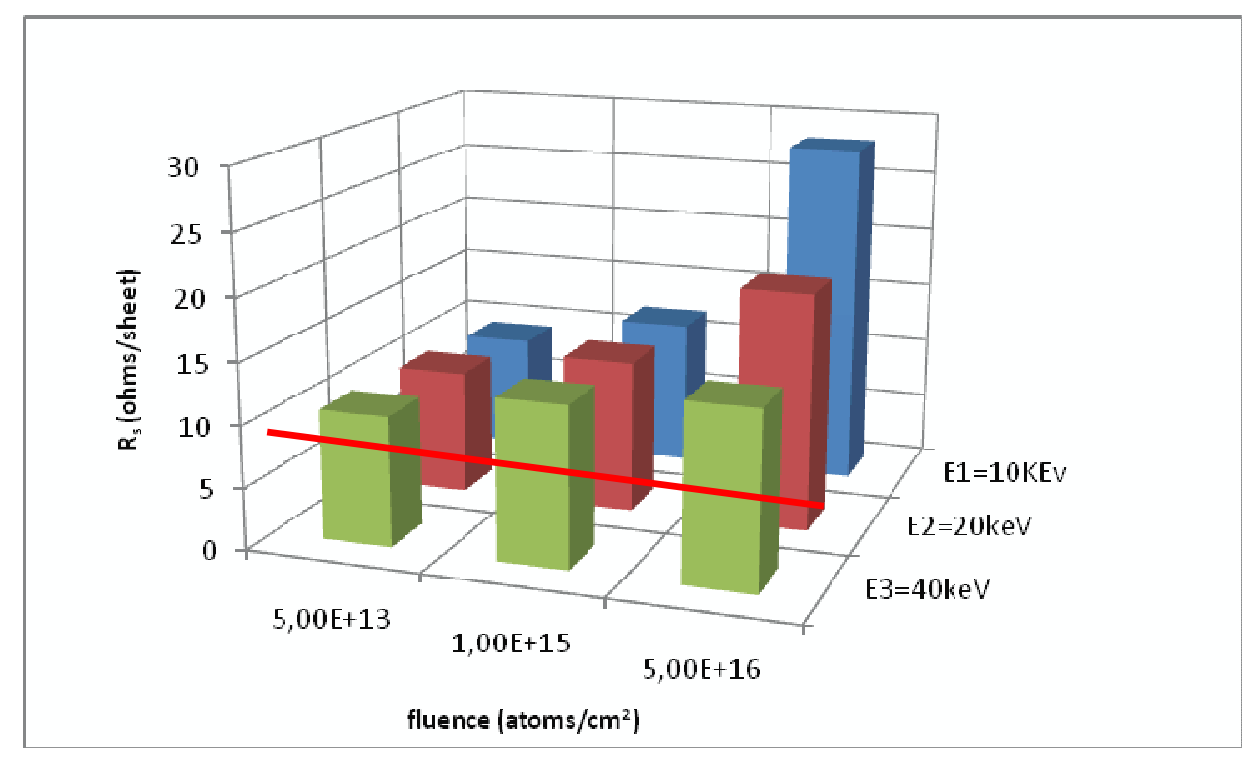

Figure 7: Four point probe measurements of film sheet resistance for $\mathrm{TiN} / \mathrm{SiO}_{2} / \mathrm{Si}$ samples modified by varying energy implantation (E1, E2, E3) and fluence (F1, F2, F3). Horizontal line corresponds to sheet resistance for reference $\operatorname{TiN}(7.7 \Omega /$ ). 


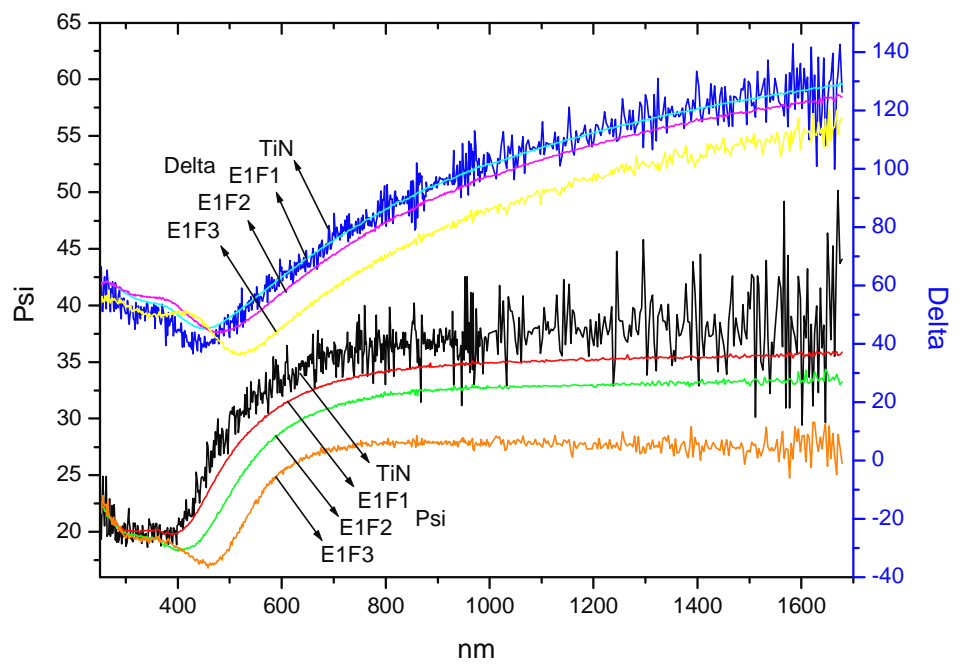

Figure 8: Spectroscopic Ellipsometry measurements of TiN/Si sample and samples irradiated at the lower E1 energy and varying fluence (F1, F2 and F3). 